\title{
Improving Diagnostic Yield of Capsule Endoscopy in Coeliac Disease: Can Flexible Spectral Imaging Colour Enhancement Play a Role?
}

\author{
Stefania Chetcuti Zammit ${ }^{\mathrm{a}}$ Mark E. McAlindon ${ }^{\mathrm{a}}$ Pierre Ellul ${ }^{\mathrm{b}}$ \\ Emanuele Rondonotti ${ }^{c}$ Cristina Carretero $^{d}$ David S. Sanders ${ }^{a}$ Reena Sidhu ${ }^{a}$ \\ ${ }^{a}$ Academic Unit of Gastroenterology, Royal Hallamshire Hospital, Sheffield, UK; ${ }^{b}$ Department of Gastroenterology, \\ Mater Dei Hospital, Msida, Malta; ' Gastroenterology Unit, Valduce Hospital, Como, Italy; ${ }^{\text {d Department of }}$ \\ Gastroenterology, University Clinic of Navarra, Pamplona, Spain
}

\section{Keywords}

Flexible spectral imaging colour enhancement $\cdot$ Small bowel capsule endoscopy · Coeliac disease

\begin{abstract}
Introduction: Flexible spectral imaging colour enhancement (FICE) is a form of virtual chromoendoscopy that is incorporated in the capsule reading software and that can be used by reviewers to enhance the delineation of lesions in the small bowel. This has been shown to be useful in the detection of pigmented (ulcers, angioectasias) lesions. However, its application to coeliac disease (CD) images from small bowel capsule endoscopies (SBCEs) has rarely been studied. Methods: This was a European, multicentre study that included 5 expert capsule reviewers who were asked to evaluate a number of normal and abnormal de-identified images from SBCEs of patients with CD to determine whether the use of FICE and blue light can improve the detection of CDrelated changes. Results: Sensitivity and specificity of conventional white light in the delineation of CD-related changes were $100 \%$. The next best image modification was FICE 1 with a sensitivity of $80 \%$ and a specificity of $100 \%$. There was no difference between conventional white light, FICE and blue light for the identification of $C D$-related changes. There was a low agreement (Fleiss kappa 0.107; $p=0.147$ ) between
\end{abstract}

\section{KARGER}

(c) 2019 S. Karger AG, Basel

karger@karger.com

www.karger.com/dig expert reviewers in selecting the best image modification that detected CD-related changes. Conclusions: FICE and blue light were not found to be superior to conventional white light in the delineation of macroscopic changes related to CD on SBCEs.

(c) 2019 S. Karger AG, Basel

\section{Introduction}

Although most guidelines on adult coeliac disease (CD) recommend gastroduodenoscopy and duodenal biopsies in patients with positive CD serology and suggestive symptoms $[1,2]$, some patients are unwilling to undergo this procedure to establish a definitive diagnosis of CD. Small bowel capsule endoscopy (SBCE) can play a useful role in this cohort of patients [3]. Even in patients undergoing a gastroduodenoscopy and duodenal biopsies, pitfalls in the diagnosis of CD still exist mainly due to the patchy nature of $\mathrm{CD}$ [4-6]. Unless at least 4 biopsy specimens (including a duodenal bulb biopsy) are taken from the duodenum $[2,4]$ and the samples are properly oriented during preparation for histological assessment [7], duodenal biopsy sampling may be sub-optimal. These factors establish a further role for SBCE in patients with a high suspicion of $\mathrm{CD}$ but negative duodenal histology,

Stefania Chetcuti Zammit

Department of Gastroenterology Royal Hallamshire Hospital

Glossop road, Sheffield S10 2JF (UK)

E-Mail stf_che@yahoo.com 
due to its panoramic underwater view that magnifies changes in the small bowel (SB) and improves delineation of lesions.

Over the years, attempts have been made to improve the detectability of pathological lesions on SCBE. One such modality is the application of flexible spectral imaging colour enhancement (FICE), a form of virtual chromoendoscopy. It can be applied to images from SBCE by adjusting settings on the RAPID reading software [8]. As the capsule travels through the SB, images in the white light spectrum are captured. Post-production computer algorithms then select single wavelength images in the red, green and blue spectra to reconstruct FICE enhanced images $[9,10]$. The aim is to improve the detection of mucosal changes such as the delineation of small blood vessels, enhance the resolution of mucosal patters and augment colour differences [11]. Blue light is an additional image-enhancing setting available on the RAPID software that enables light in the wavelength range of $490-430 \mathrm{~nm}$ to be picked up from white light images.

Macroscopic changes of CD occur secondary to different degrees of villous atrophy resulting in scalloping of folds, fissuring and mosaic pattern of mucosa. Other features include complete absence of villi giving the appearance of villous atrophy. Ulcers can also be present at times $[12,13]$. These changes can be identified on conventional white light SBCE. However, the detection of findings can be challenging due to subtle changes only affecting the duodenal bulb in ultra-short CD [14]. The manifestation of $\mathrm{CD}$ is also often patchy in nature [6]. Image modification to enhance features of $\mathrm{CD}$ may help the delineation of CD-related changes particularly for novice SBCE reviewers. There is only one study that reports on the use of FICE for the detection of changes related to CD in the SB. However, only one macroscopic feature - villous atrophy was studied and the same cohort included patients with other pathologies apart from CD [15]. Our study is the first to report on the utility of FICE and blue light in a cohort of patients with CD alone on SBCE.

The sensitivity of SBCE in detecting CD-related changes is reported to be as low as $70 \%$ in some studies [16]. The sensitivity of SBCE depends on the reviewer's prestudy experience declining when the reviewer's pre-study experience is low [16]. Changes related to CD in the SB can vary from very mild to more severe features. Subtle changes can contribute to decreasing the sensitivity of SBCE to detect CD-related changes. The aim of this study was to assess whether there is any additional benefit in using FICE or blue light over conventional white light for the detection of changes related to $\mathrm{CD}$ on SBCE.

\section{Methods}

Patients and Methods

This was a multicentre, European study that included 5 expert capsule endoscopy reviewers (>300 capsules/year). They were asked to evaluate a number of de-identified images from SBCEs of patients with confirmed CD on duodenal histology to determine whether the use of FICE and blue light can improve the detection of CD-related changes on SBCE. Features of CD on SBCE images and normal SBCE images were initially identified and features of CD confirmed by 2 expert SBCE reviewers (>300 capsules/year). These 2 expert reviewers involved in the initial preparation of the images were not involved in the actual study. Findings on these images were then set as the standard to which results from each reviewer were compared to. Features of CD included: (1) scalloping of the mucosa, (2) fissuring of folds, (3) mosaic pattern, (4) villous atrophy, (5) nodularity of mucosa. The reviewers were blinded to each other's findings and to the histological Marsh classification of disease.

In the first part of the study, the reviewers were asked to examine a set of 50 images consisting of both normal (25) SB images and 25 images showing CD-related changes. Conventional white light, FICE I, II, III and blue light were represented in 1 question each in this section. Each question consisted of 5 normal and 5 abnormal images from the SBCE of the same patient (Fig. 1). The reviewers were asked to pick up abnormal images (Which images show features of $C D$ ?). Depending on the number of abnormal images correctly picked up by each reviewer, the sensitivity and specificity of conventional white light, different FICE settings and blue light were calculated.

In the second part of the study, reviewers were asked to go through 55 abnormal images (11 questions) and to compare between conventional white light, different FICE (I, II, III) settings and blue light as the best modality to delineate changes of CD on SBCE. Each question consisted of abnormal images in conventional white light, different FICE settings or blue light (Fig. 2). Images showed either moderate (5 questions), severe features of CD (5 questions) or ulcers (1 question). Severity of features on each image were pre-determined by an expert SBCE reviewer (>300 capsules/year) before the commencement of this study depending on the presence of patchy/continuous pattern and prominence of lesions. This was confirmed by a second SBCE reviewer (>300 capsules/year). The interobservor agreement between reviewers was then calculated.

\section{Ethics Approval}

The study protocol was approved by the Yorkshire and Humber Research Ethics Committee (IRAS 232382) and registered with the local research and development department of Sheffield Teaching Hospital NHS Foundation Trust under the registration number STH 19998. All images used in this study were de-identified. No additional consent was required for the study with the use of de-identified videos as assessed and approved formally by the Research Ethics Committee.

\section{Statistical Analysis}

IBM SPSS Statistics version 23 and Microsoft Excel for Mac version 16.16.4 were used to analyse the data. Frequencies of choice were calculated for each expert reviewer. Fleiss' kappa coefficient $(\mathrm{K})$ was used to measure the degree of agreement amongst the 5 reviewers. Agreement according to $\mathrm{K}$ value was considered 

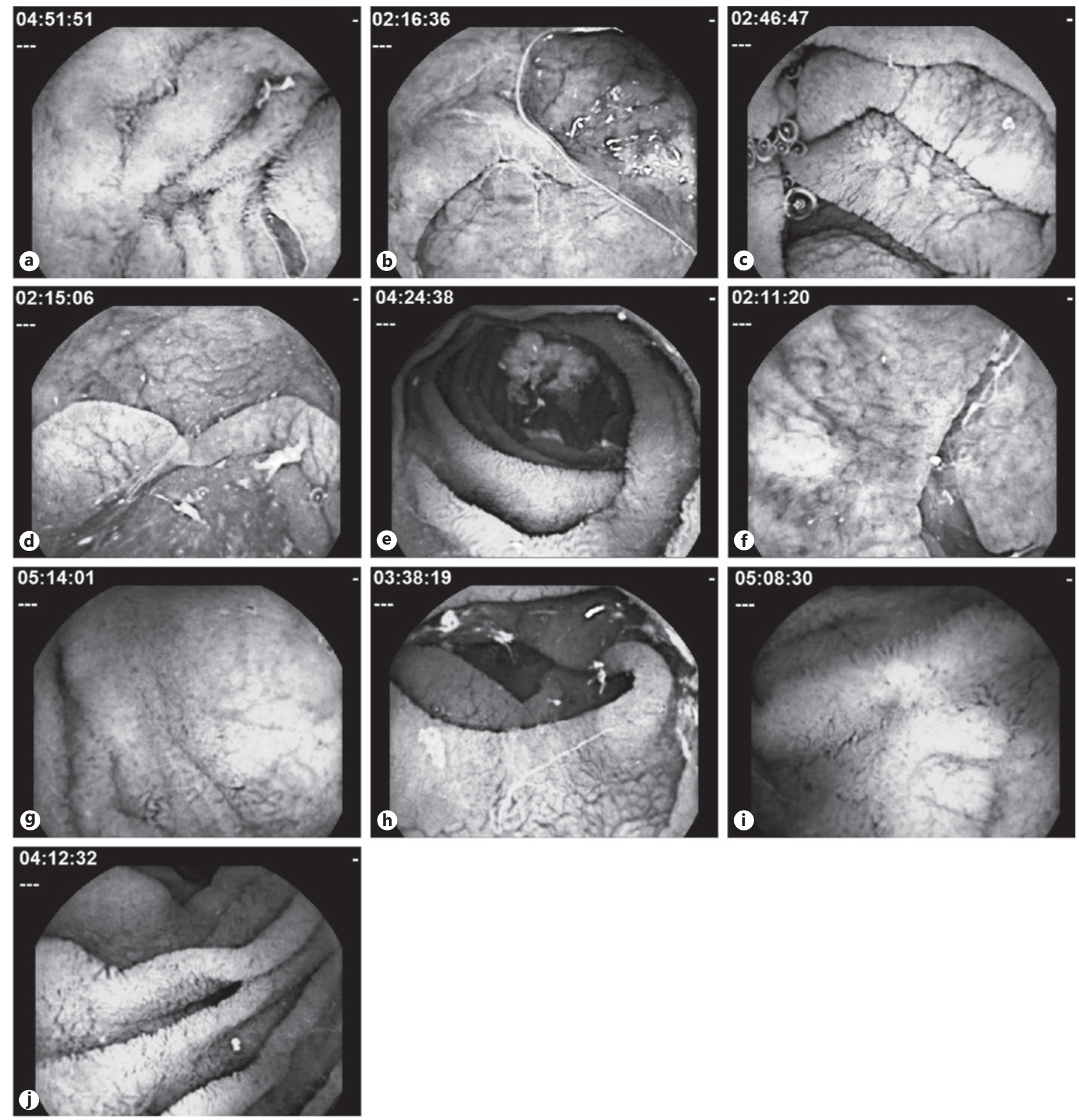

Fig. 1. Images on SBCE modified by FICE $2(\mathbf{b}-\mathbf{d}, \mathbf{f}, \mathbf{h})$ show features of $C D(\mathbf{a}, \mathbf{e}, \mathbf{g}, \mathbf{i}, \mathbf{j})$ are normal images.

as follows: $<0$ indicated poor agreement, $0.00-0.20$ slight agreement, $0.21-0.40$ fair agreement, $0.41-0.60$ moderate agreement, $0.61-0.80$ substantial agreement, and $0.81-1.00$ almost perfect agreement [17]. A significance level of $>0.05(p>0.05)$ meant that there was not enough evidence to conclude that the reviewers' agreement was different from what would be expected to be achieved by chance.

Contingency tables were also constructed to calculate the sensitivity and specificity of conventional white light, different FICE settings and blue light. 

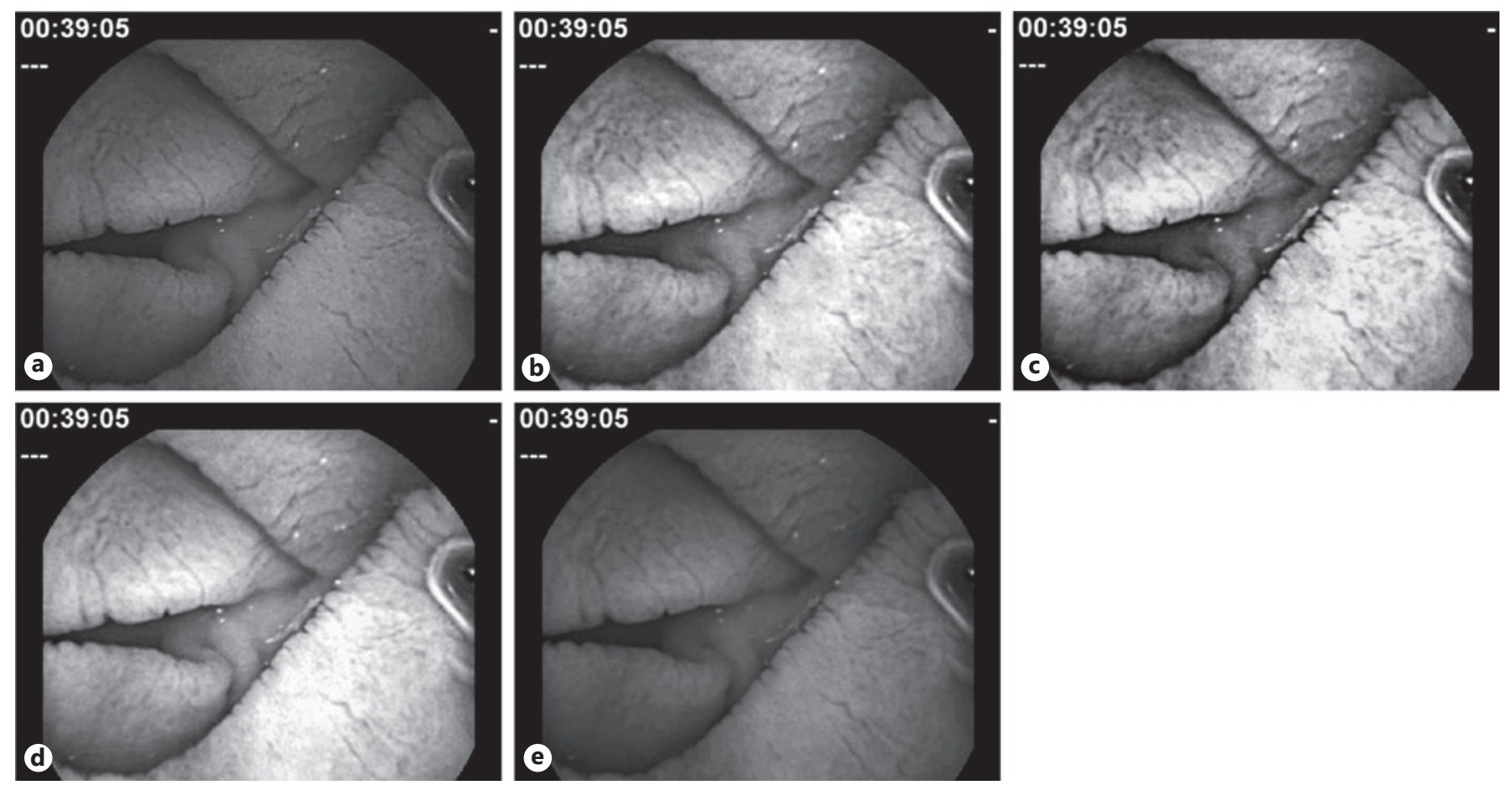

Fig. 2. Fissuring of mucosa in a patient with CD on SBCE: (a) conventional white light; (b) FICE 1; (c) FICE 2; (d) FICE 3; (e) blue light.

\section{Power Calculation}

Currently, the sensitivity of SBCE in detecting CD-related changes using conventional white light, varies between 70 and $93 \%$ $[3,16,18-22]$. To improve the sensitivity of SBCE from the lowest recorded value of $70.0-93.0 \%$ by the introduction of FICE on reviewing SBCE images, (using G Power) it was estimated that a sample size of 28 would be needed to correctly identify changes of CD with $95 \%$ power and a 5\% 2-sided significance level. We have exceeded the required number to reach the $95 \%$ power in both sections of the study.

Our study is the first to report on the utility of FICE and blue light in a cohort of patients with CD alone on SBCE.

\section{Results}

All patients had duodenal atrophy (Marsh 3a: $18.8 \%$ $[n=3]$, Marsh 3b: 50\% [ $n=8]$, Marsh 3c: $31.3 \%$ [ $n=5])$ on biopsies taken from the second part of the duodenum. All reviewers identified the abnormal images in conventional white light resulting in $100 \%$ sensitivity and specificity. FICE 1 had the next best sensitivity in the identification of abnormal images followed by sensitivities for FICE 2 and 3. Blue light had the lowest sensitivity in detecting features of CD (Table 1).

Although FICE 1 and 2 were the most popular settings chosen by reviewers to delineate changes of $\mathrm{CD}$ in the second section, there was no statistically significant difference between reviewers ( $p=0.193$; Table 2). The low overall $\mathrm{K}(\mathrm{K} 0.107 ; p=0.147)$ was consistent with poor correlation of the preferred modality amongst different expert reviewers (Table 3 ). The overall $\mathrm{K}$ were similarly low for moderate $(K=0.107 ; p=0.147)$ and severe $(\mathrm{K}=0.107 ; p=0.147)$ changes of $\mathrm{CD}$. The $\mathrm{K}$ was low even when considered separately for different light settings and it was not statistically significant in all cases (conventional white light $\mathrm{K}=-0.122, p=1.801$, FICE $1 \mathrm{~K}=0.189$, $p=0.148$, FICE $2 \mathrm{~K}=0.149, p=0.255)$. None of the reviewers favoured FICE 3 and blue light. Therefore, these have been left out of Tables 1 and 2. This also means that the K could not be calculated for FICE 3 and blue light.

\section{Discussion}

This study confirms that FICE and blue light do not have any additional benefit in helping the delineation of $\mathrm{CD}$ changes on SBCE when compared to conventional white light.

Dye chromoendoscopy has been utilised in the context of IBD surveillance to help the visualisation of subtle lesions and to define surface staining patterns enabling 
Table 1. Mean sensitivity, specificity, positive and negative likelihood ratios for normal light, FICE and blue light

\begin{tabular}{|c|c|c|c|c|c|}
\hline Reviewer 1 & 100 & 80 & 60 & 0 & 40 \\
\hline Reviewer 2 & 100 & 80 & 100 & 100 & 60 \\
\hline Reviewer 3 & 100 & 80 & 60 & 60 & 20 \\
\hline Reviewer 4 & 100 & 60 & 60 & 40 & 20 \\
\hline Reviewer 5 & 100 & 100 & 100 & 100 & 100 \\
\hline Mean sensitivity & 100 & 80 & 76 & 60 & 48 \\
\hline Reviewer 2 & 100 & 100 & 100 & 100 & 100 \\
\hline Reviewer 3 & 100 & 100 & 100 & 100 & 100 \\
\hline Reviewer 4 & 100 & 100 & 80 & 60 & 80 \\
\hline Reviewer 5 & 100 & 100 & 100 & 100 & 100 \\
\hline Mean specificity & 100 & 100 & 96 & 92 & 96 \\
\hline
\end{tabular}

FICE, flexible spectral imaging colour enhancement.

Table 2. Favoured modality of normal light or different FICE settings by all the 5 reviewers

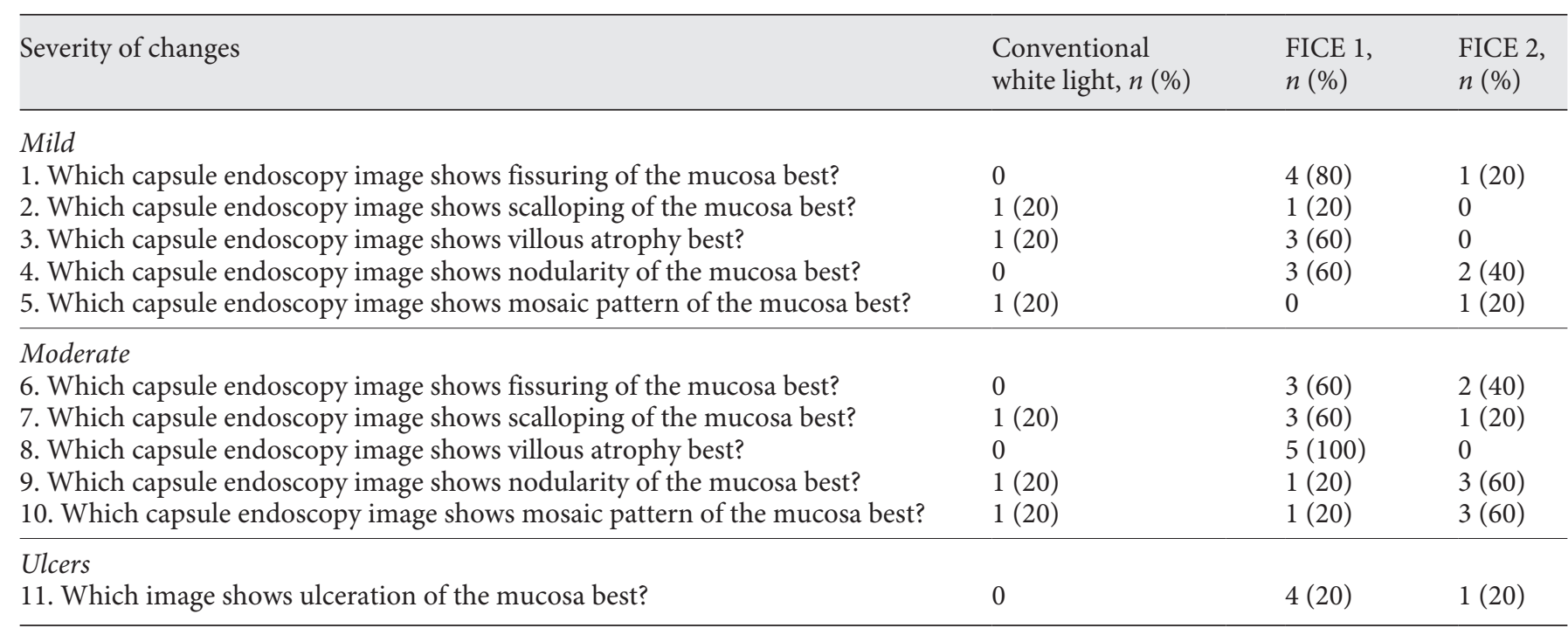

FICE, flexible spectral imaging colour enhancement.

targeted biopsies to be taken [23]. Virtual chromoendoscopy has been applied to identifying adenomatous polyps and areas of dysplasia during upper GI endoscopy and in helping the identification of polyps during colonoscopy $[24,25]$. It has also been shown to be useful in detecting high-grade dysplasia in Barrett's epithelium [26].

The use of FICE to aid reporting of SBCE has been widely debated in the context of SB ulcers and vascular lesions. FICE can improve the detection of ulcerative lesions by highlighting inflammatory halos and increasing the contrast between pathological areas and surrounding mucosa $[8,15,27-31]$. Whilst some early studies showed that FICE performed better than conventional white light for the detection of SB ulcers, other studies have contradicted this finding [32].

FICE has also been studied for the detection of angioectasias with some studies showing benefit $[8,15,28-30$, 32,33 . This is because it improves the detection of light in certain spectrums that is absorbed by haemoglobin. In 2 studies on patients with obscure gastrointestinal bleeding, patients with negative SBCEs in standard view were enrolled. FICE was able to detect significant lesions in 
Table 3. Fleiss' kappa co-efficient for normal light and FICE settings

\begin{tabular}{lrrrr}
\hline & Total & $\begin{array}{l}\text { Conventional } \\
\text { white light }\end{array}$ & FICE 1 & FICE 2 \\
\hline $\mathrm{K}$ & 0.107 & -0.122 & 0.189 & 0.149 \\
$\mathrm{SE}$ & 0.073 & 0.095 & 0.129 & 0.129 \\
$\mathrm{Z}$ & 1.451 & -1.284 & 1.447 & 1.151 \\
$\mathrm{P}$ & 0.147 & 1.801 & 0.148 & 0.250 \\
Lower & -0.037 & -0.309 & -0.066 & -0.104 \\
Upper & 0.250 & 0.064 & 0.440 & 0.402 \\
\hline
\end{tabular}

FICE, flexible spectral imaging colour enhancement; SE, standard error.

$5.8-21 \%$ of patients $[34,35]$. However, FICE has been shown to have a high false-positive rate in the delineation of angioectasias [36].

This is the first and largest study on the use of FICE and blue light to help delineate changes related to CD on SBCE. In this study, experts were asked to determine which FICE setting, blue light or conventional white light was the best modality to detect mucosal abnormalities. Both FICE and blue light were inferior to conventional white light in detecting CD changes on SBCE images. The calculation of sensitivity and specificity of FICE add further to the current evidence on FICE when compared to conventional white light. The sensitivity and specificity of detecting SB pathologies using FICE were similar to conventional white light in a study by Kobayashi et al. [37]. Similarly, the sensitivity and specificity of FICE in detecting CD-related lesions in this study were similar in the case of FICE 1,2 , and 3 but inferior to conventional white light.

The low K co-efficient confirms the lack of agreement between reviewers to favour any FICE settings or blue light in the detection CD changes on SBCE. K co-efficient was low irrespective of the severity of CD changes on SBCE suggesting that FICE will not help increase the detection of CD changes on SBCE even if the changes are subtle.

Our findings on the use of FICE on SBCE images to help detect features of $\mathrm{CD}$ reflect literature previously published on the use of dye chromoendoscopy during gastroduodenoscopy to improve the detection of macroscopic features of CD [38]. In the study by Johnston et al. [38], chromoendoscopy identified an additional number of patients with CD (54\% with chromoendoscopy vs. $42 \%$ on normal endoscopy) on gastroduodenoscopy. However, the sensitivity of chromoendoscopy was by far inferior to the sensitivity of CD serology ( $89 \%$ anti-tissue transglutaminase antibody, $78 \%$ for anti-endomysial anti- body). Thus, it was concluded that chromoendoscopy could not be recommended for routine clinical practice during gastroduodenoscopy in patients with suspected and established CD.

One limitation of this study is the exclusion of mild macroscopic CD-related changes due to the assumption that these would have been much harder to delineate, thus impacting negatively on the degree of agreement between expert reviewers. It was assumed that all the expert reviewers had the same pre-study experience of reviewing SBCEs from patients with CD. Inclusion of a section prior to this study, with images to ensure familiarisation of expert reviewers with macroscopic CD-related changes on SBCEs might have resulted in an improvement in the agreement between reviewers.

In a recent meta-analysis, the delineation of SB pathologies using FICE was studied [39]. The meta-analysis included 3 studies that assessed the improvement in delineation of lesions and 5 studies that evaluated the detection of lesions. Overall, FICE did not help to improve the detection of SB lesions except for pigmented lesions where FICE performed better. FICE 1 setting improved the delineation of $89 \%$ of angioectasias and $45 \%$ of ulcers and erosions. The recently published ESGE technical guidelines [40] do not recommend the routine use of virtual chromoendoscopy during SBCE as this does not improve the delineation of lesions. This statement mainly refers to vascular and ulcerative lesions, since studies referring to images taken from SBCE of patients with CD are lacking.

Only one group of authors has studied the role of FICE in the detection of CD-related changes. Two gastroenterologists, blinded to each other's results, were asked to rank the quality of delineation of SB pathologies as better, equivalent, or worse than conventional white light. FICE 1 and 2 improved the detection of villous atrophy with a high K between expert reviewers [15]. There are considerable differences between the way this study was conducted and our methodology. Cotter et al. [15] included other SB pathologies in their study apart from CD-related changes including angioectasias and ulcers unlike in our case where the main focus was on CD. Cotter et al. [15] only considered oedema and villous atrophy but did not assess the effect of FICE on the delineation of other macroscopic features of $\mathrm{CD}$. They also asked reviewers to grade FICE images as better, equivalent or worse compared to conventional white light unlike in our case where a comparison of different FICE settings and conventional white light was done for each question. Having only 2 images to choose from might explain the much higher $\mathrm{K}$ values obtained in this study unlike in our study where 
the agreement between reviewers was very poor. In addition, a greater number of expert reviewers were included in our study.

Narrowing the bandwidth with FICE enhances the hypervascularity of lesions. Most of the macroscopic changes of $\mathrm{CD}$ occur due to various degrees of villous shorting and not due to vascular changes [41]. This can explain the lack of additional benefit of FICE on SBCE in patients with CD.

Data on the use of FICE and blue light are very sparse and at times contradictory. Some studies evaluate the use of FICE on multiple pathologies that can exist in the SB resulting in evaluation of virtual chromoendoscopy in even smaller groups of patients. Authors have used different methodologies to evaluate the use of FICE, making it harder to compare results between studies and questioning further the utility of FICE.

Despite the negative results of this study, the results are useful as they add to the limited literature that is available on the use of FICE on SBCE in suspected CD. They provide evidence that there is no additional benefit for gastroenterologists to re-review SBCE in different FICE settings or blue light after reviewing the SBCE using conventional white light when the suspicion of $\mathrm{CD}$ is high. The use of FICE will not help in the detection of subtle CDrelated changes by novice SBCE reviewers.

\section{Conclusions}

This study has demonstrated that amongst 5 expert SBCE reviewers, FICE settings and blue light were not better than conventional white light in the identification of macroscopic changes of CD. Reviewing SBCE images with different FICE settings where CD is suspected will not help to delineate these changes better.

\section{Statement of Ethics}

The study protocol was approved by the Yorkshire and Humber Research Ethics Committee (IRAS 232382) and registered with the local research and development department of Sheffield Teaching Hospital NHS Foundation Trust under the registration number STH 19998.

\section{Disclosure Statement}

The authors have no conflicts of interest to declare.

\section{Funding Sources}

None.

\section{References}

1 Downey L, Houten R, Murch S, Longson D; Guideline Development Group. Recognition, assessment, and management of coeliac disease: summary of updated NICE guidance. BMJ. 2015 Sep;351:h4513.

2 Ludvigsson JF, Bai JC, Biagi F, Card TR, Ciacci C, Ciclitira PJ, et al.; BSG Coeliac Disease Guidelines Development Group; British Society of Gastroenterology. Diagnosis and management of adult coeliac disease: guidelines from the British Society of Gastroenterology. Gut. 2014 Aug;63(8):1210-28.

3 Hopper AD, Sidhu R, Hurlstone DP, McAlindon ME, Sanders DS. Capsule endoscopy: an alternative to duodenal biopsy for the recognition of villous atrophy in coeliac disease? Dig Liver Dis. 2007 Feb;39(2):140-5.

4 Pais WP, Duerksen DR, Pettigrew NM, Bernstein CN. How many duodenal biopsy specimens are required to make a diagnosis of celiac disease? Gastrointest Endosc. 2008 Jun; 67(7):1082-7.

5 Green PH. Celiac disease: how many biopsies for diagnosis? Gastrointest Endosc. 2008 Jun; 67(7):1088-90.

6 Hopper AD, Cross SS, Sanders DS. Patchy villous atrophy in adult patients with suspected gluten-sensitive enteropathy: is a multiple du- odenal biopsy strategy appropriate? Endoscopy. 2008 Mar;40(3):219-24.

7 Collin P, Kaukinen K, Vogelsang H, Korponay-Szabó I, Sommer R, Schreier E, et al. Antiendomysial and antihuman recombinant tissue transglutaminase antibodies in the diagnosis of coeliac disease: a biopsyproven European multicentre study. Eur J Gastroenterol Hepatol. 2005 Jan;17(1):8591.

8 Sato Y, Sagawa T, Hirakawa M, Ohnuma H, Osuga T, Okagawa Y, et al. Clinical utility of capsule endoscopy with flexible spectral imaging color enhancement for diagnosis of small bowel lesions. Endosc Int Open. 2014 Jun;2(2):E80-7.

9 Fedeli P, Gasbarrini A, Cammarota G. Spectral endoscopic imaging: the multiband system for enhancing the endoscopic surface visualization. J Clin Gastroenterol. 2011 Jan; 45(1):6-15.

10 Togashi K, Osawa H, Koinuma K, Hayashi Y, Miyata T, Sunada K, et al. A comparison of conventional endoscopy, chromoendoscopy, and the optimal-band imaging system for the differentiation of neoplastic and non-neoplastic colonic polyps. Gastrointest Endosc. 2009 Mar;69(3 Pt 2):734-41.
11 Pohl J, May A, Rabenstein T, Pech O, Ell C. Computed virtual chromoendoscopy: a new tool for enhancing tissue surface structures. Endoscopy. 2007 Jan;39(1):80-3.

12 Tursi A, Brandimarte G, Giorgetti GM, Gigliobianco A. Endoscopic features of celiac disease in adults and their correlation with age, histological damage, and clinical form of the disease. Endoscopy. 2002 Oct;34(10):78792.

13 Biagi F, Rondonotti E, Campanella J, Villa F, Bianchi PI, Klersy C, De Franchis R, Corazza GR. Video capsule endoscopy and histology for small-bowel mucosa evaluation: a comparison performed by blinded observers. Clin Gastroenterol Hepatol. 2006 Aug;4(8):9981003.

14 Mooney PD, Kurien M, Evans KE, Rosario E, Cross SS, Vergani P, et al. Clinical and Immunologic Features of Ultra-Short Celiac Disease. Gastroenterology. 2016 May; 150(5): 1125-34.

15 Cotter J, Magalhães J, de Castro FD, Barbosa M, Carvalho PB, Leite S, et al. Virtual chromoendoscopy in small bowel capsule endoscopy: new light or a cast of shadow? World J Gastrointest Endosc. 2014 Aug; 6(8):359-65. 
16 Petroniene R, Dubcenco E, Baker JP, Ottaway CA, Tang SJ, Zanati SA, et al. Given capsule endoscopy in celiac disease: evaluation of diagnostic accuracy and interobserver agreement. Am J Gastroenterol. 2005 Mar;100(3): 685-94.

17 Landis JR, Koch GG. The measurement of observer agreement for categorical data. Biometrics. 1977 Mar;33(1):159-74.

18 El-Matary W, Huynh H, Vandermeer B. Diagnostic characteristics of given video capsule endoscopy in diagnosis of celiac disease: a meta-analysis. J Laparoendosc Adv Surg Tech A. 2009 Dec;19(6):815-20.

19 Luján-Sanchis M, Pérez-Cuadrado-Robles E, García-Lledó J, Juanmartiñena Fernández JF, Elli L, Jiménez-García VA, et al. Role of capsule endoscopy in suspected celiac disease: A European multi-centre study. World J Gastroenterol. 2017 Jan;23(4):703-11.

20 Murray JA, Rubio-Tapia A, Van Dyke CT, Brogan DL, Knipschield MA, Lahr B, et al. Mucosal atrophy in celiac disease: extent of involvement, correlation with clinical presentation, and response to treatment. Clin Gastroenterol Hepatol. 2008 Feb;6(2):186-93; quiz 125.

21 Rokkas T, Niv Y. The role of video capsule endoscopy in the diagnosis of celiac disease: a meta-analysis. Eur J Gastroenterol Hepatol. 2012 Mar;24(3):303-8.

22 Rondonotti E, Spada C, Cave D, Pennazio M, Riccioni ME, De Vitis I, et al. Video capsule enteroscopy in the diagnosis of celiac disease: a multicenter study. Am J Gastroenterol. 2007 Aug;102(8):1624-31.

23 Sekra A, Schauer C, Mills L, Vandal AC, Rose $\mathrm{T}$, Lal D, et al. Chromoendoscopy versus standard colonoscopy for detection of nonpolypoid dysplasia in patients with inflammatory bowel disease. N Z Med J. 2018 Jul;131(1478): 32-8.

24 Lami G, Galli A, Macrì G, Dabizzi E, Biagini MR, Tarocchi M, et al. Gastric and duodenal polyps in familial adenomatous polyposis patients: conventional endoscopy vs virtual chromoendoscopy (fujinon intelligent color enhancement) in dysplasia evaluation. World J Clin Oncol. 2017 Apr;8(2): 168-77.
25 Akarsu C, Sahbaz NA, Dural AC, Kones O, Binboga S, Kabuli HA, et al. FICE in Predicting Colorectal Flat Lesion Histology. JSLS. 2017 Oct-Dec;21(4).pii:e2017.00050.

26 Camus M, Coriat R, Leblanc S, Brezault C, Terris B, Pommaret E, et al. Helpfulness of the combination of acetic acid and FICE in the detection of Barrett's epithelium and Barrett's associated neoplasias. World J Gastroenterol. 2012 Apr;18(16):1921-5.

27 Konishi M, Shibuya T, Mori H, Kurashita E, Takeda T, Nomura O, et al. Usefulness of flexible spectral imaging color enhancement for the detection and diagnosis of small intestinal lesions found by capsule endoscopy. Scand J Gastroenterol. 2014 Apr;49(4):501-

28 Nogales Rincón O, Merino Rodríguez B, González Asanza C, Fernández-Pacheco PM. [Utility of capsule endoscopy with flexible spectral imaging color enhancement in the diagnosis of small bowel lesions]. Gastroenterol Hepatol. 2013 Feb;36(2):63-8.

29 Sakai E, Endo H, Kato S, Matsuura T, Tomeno $\mathrm{W}$, Taniguchi L, et al. Capsule endoscopy with flexible spectral imaging color enhancement reduces the bile pigment effect and improves the detectability of small bowel lesions. BMC Gastroenterol. 2012 Jul;12(1):83.

30 Duque G, Almeida N, Figueiredo P, Monsanto P, Lopes S, Freire P, et al. Virtual chromoendoscopy can be a useful software tool in capsule endoscopy. Rev Esp Enferm Dig. 2012 May;104(5):231-6.

31 Rimbaş M, Negreanu L, Ciobanu L, Benguş A, Spada C, Băicus CR, et al. Is virtual chromoendoscopy useful in the evaluation of subtle ulcerative small-bowel lesions detected by video capsule endoscopy? Endosc Int Open. 2015 Dec;3(6):E615-20.

32 Boal Carvalho P, Magalhães J, Dias de Castro F, Gonçalves TC, Rosa B, Moreira MJ, et al. Virtual chromoendoscopy improves the diagnostic yield of small bowel capsule endoscopy in obscure gastrointestinal bleeding. Dig Liver Dis. 2016 Feb;48(2):172-5.

33 Imagawa $\mathrm{H}$, Oka $\mathrm{S}$, Tanaka $\mathrm{S}$, Noda I, Higashiyama M, Sanomura Y, et al. Improved detectability of small-bowel lesions via capsule endoscopy with computed virtual chro- moendoscopy: a pilot study. Scand J Gastroenterol. 2011 Sep;46(9):1133-7.

34 Minami-Kobayashi Y, Yamada A, Watabe H, Suzuki H, Hirata Y, Yamaji Y, et al. Efficacy of repeat review with flexible spectral imaging color enhancement in patients with no findings by capsule endoscopy. Saudi J Gastroenterol. 2016 Sep-Oct;22(5):385-90.

35 Dias de Castro F, Magalhães J, Boal Carvalho P, Cúrdia Gonçalves T, Rosa B, Moreira MJ, et al. Improving diagnostic yield in obscure gastrointestinal bleeding-how virtual chromoendoscopy may be the answer. Eur J Gastroenterol Hepatol. 2015 Jun;27(6):735-40.

36 Nakamura M, Ohmiya N, Miyahara R, Ando $\mathrm{T}$, Watanabe O, Kawashima H, et al. Usefulness of flexible spectral imaging color enhancement (FICE) for the detection of angiodysplasia in the preview of capsule endoscopy. Hepatogastroenterology. 2012 Jul-Aug; 59(117):1474-7.

37 Kobayashi Y, Watabe H, Yamada A, Hirata Y, Yamaji Y, Yoshida H, et al. Efficacy of flexible spectral imaging color enhancement on the detection of small intestinal diseases by capsule endoscopy. J Dig Dis. 2012 Dec;13(12): $614-20$

38 Johnston AJ, Kurien M, Avgerinos A, Mooney $\mathrm{PD}$, Sanders DS. Is there a role for chromoendoscopy in the diagnosis of coeliac disease? J Gastrointestin Liver Dis. 2014 Mar;23(1): 103-4.

39 Yung DE, Boal Carvalho P, Giannakou A, Kopylov U, Rosa B, Rondonotti E, et al. Clinical validity of flexible spectral imaging color enhancement (FICE) in small-bowel capsule endoscopy: a systematic review and meta-analysis. Endoscopy. 2017 Mar;49(3):258-69.

40 Rondonotti E, Spada C, Adler S, May A, Despott EJ, Koulaouzidis A, et al. Small-bowel capsule endoscopy and device-assisted enteroscopy for diagnosis and treatment of smallbowel disorders: European Society of Gastrointestinal Endoscopy (ESGE) Technical Review. Endoscopy. 2018 Apr;50(4):423-46.

41 Pohl J, Aschmoneit I, Schuhmann S, Ell C. Computed image modification for enhancement of small-bowel surface structures at video capsule endoscopy. Endoscopy. 2010 Jun; 42(6):490-2. 